\section{P2-187 MENTAL HEALTH AND SMOKING AMONG ADOLESCENTS FROM A COHORT IN SOUTHERN BRAZIL}

doi:10.1136/jech.2011.142976j.22

A M Menezes, * S Dumith, J Martinez, A E Silva, A Cascaes, F Ferreira, G Dominguez, G França, J Damé, K Ngale, L Anselmi. Federal University of Pelotas, Pelotas, Rio Grande do Sul, Brazil

Background Smoking is one of the major risk factors for various diseases. There is some evidence that smoking is associated with mental health problems.

Objective To evaluate the association among mental health problems and smoking in teenagers at 15 years old in a population-based birth cohort.

Methods 4325 adolescents from the 1993 birth cohort, from the city of Pelotas, southern Brazil, were examined. Smoking was defined as to have smoked one or more cigarettes in the previous 30 days. Mental health was assessed according to the total score of the questionnaire Strengths and Difficulties Questionnaire being considered as positive when the score was $\geq 20$ points. Data were analysed using Poisson regression with adjustment for robust variance.

Results Smoking prevalence was $6.0 \%$ and about $30 \%$ of the adolescents presented any problem related to mental health. In the crude analysis, the prevalence ratio to present mental health problem was 3.3 (95\% CI 2.5 to 4.2 ) and after the adjusted analysis (for sex, age, skin colour, family income, maternal education, smoking among friends, employment in the last year, school failure, physical activity at leisure and experimental use of alcohol) it decreased to 1.9 (95\% CI 1.2 to 2.3) among smokers compared to non-smokers.

Conclusion We concluded that mental health problems in adolescence may be related to tobacco consumption.

\section{P2-188 PROSPECTIVE STUDY OF ALBUMINURIA ON CARDIOVASCULAR DISEASE MORTALITY AND MORBIDITY IN THE GENERAL POPULATION}

doi:10.1136/jech.2011.142976j.23

P Metcalf, ${ }^{*}$ R Scragg. University of Auckland, Auckland, New Zealand

Introduction Albuminuria predicts cardiovascular disease (CVD) in people with diabetes mellitus. However, few studies have been carried out in the general population.

Methods A total of 5260 participants aged $\geq 40$ years from worksites in New Zealand were interviewed between 1988 and 1990. Participants were followed for up to 19 years. First CVD events were defined using ICD-9 and ICD-10 coding. Early morning urinary albumin levels of $30-300 \mathrm{mg} / \mathrm{l}$ were classified as microalbuminuric $(n=249)$ and those above $300 \mathrm{mg} / \mathrm{l}$ as proteinuric $(n=23)$. HRs were calculated after adjusting for age, gender and ethnicity in the total population and after excluding participants with new or previously diagnosed diabetes $(n=5010)$.

Results Microalbuminuria was associated with increased hazards rates for all CVD events, all-cause mortality, CVD morbidity and mortality in the total population and after excluding participants with diabetes (Abstract P2-188 table 1). Similarly, proteinuria was only associated with increased HRs for all CVD events and mortality (Abstract P2-188 table 1). HRs remained significant after further adjusting for body mass index, lipids and hypertension.
Abstract P2-188 Table 1 HRs $(95 \% \mathrm{CI})$

\begin{tabular}{lllll}
\hline & $\begin{array}{l}\text { Microalbuminuria } \\
\text { Total population }\end{array}$ & Proteinuria & $\begin{array}{l}\text { Microalbuminuria } \\
\text { Non-diabetic population }\end{array}$ & Proteinuria \\
\hline All CVD events & $1.73(1.38$ to & $2.30(1.19$ to & $1.64(1.25$ to & $3.08(1.5$ to \\
& $2.18)^{* * *}$ & $4.43)^{*}$ & $2.14)^{* * *}$ & $6.2)^{* *}$ \\
All deaths & $1.96(1.50$ to & $1.23(0.40$ to & $1.67(1.21$ to & $2.03(0.63$ to \\
& $2.54)^{* * *}$ & $3.83)$ & $2.31)^{* *}$ & $6.31)$ \\
CVD morbidity & $1.66(1.29$ to & $2.57(1.33$ to & $1.52(1.13$ to & $3.40(1.7$ to \\
& $2.14)^{* * *}$ & $2.14)^{* *}$ & $2.05)^{* *}$ & $6.84)^{* * *}$ \\
CVD mortality & $2.86(1.70$ to & & $2.66(1.44$ to & \\
& $4.81)^{* * *}$ & & $4.93)^{* *}$ & \\
\hline
\end{tabular}

${ }^{*} 0.01<\mathrm{p}<0.05 ;{ }^{* *} 0.001<\mathrm{p}<0.01 ;{ }^{* * *} \mathrm{p}<0.001$

Conclusion Microalbuminuria was associated with all-cause mortality and CVD morbidity and mortality in both the general population and in non-diabetic participants and may represent generalised vascular damage.

\section{P2-189 PREVALENCE AND PATTERNS OF ALCOHOL CONSUMPTION IN CHINESE MEN AND WOMEN: THE KADOORIE BIOBANK STUDY OF 0.5 MILLION PEOPLE IN CHINA}

doi:10.1136/jech.2011.142976j.24

${ }^{1}$ I Millwood, ${ }^{*} \mathrm{~L}$ Lee, ${ }^{1} \mathrm{~L}$ Yang, ${ }^{2} \mathrm{G}$ Yu, ${ }^{1} \mathrm{R}$ Collins, ${ }^{1} \mathrm{~S}$ Lewington, ${ }^{3} \mathrm{~J}$ Chen, ${ }^{1} \mathrm{R}$ Peto, ${ }^{1} Z$ Chen. ${ }^{1}$ University of Oxford, Oxford, UK; ${ }^{2}$ Chinese Academy of Medical Sciences, Beijing, China; ${ }^{3}$ China Centre for Disease Control and Prevention, Beijing, China

Introduction Diet, lifestyle and disease patterns vary greatly from one part of China to another. Data on the prevalence and patterns of alcohol consumption in different parts of China are limited.

Methods Self-reported questionnaire data from the Kadoorie Biobank Study were used to describe the prevalence and patterns of alcohol consumption in 510000 men and women aged 30-79 years, recruited during 2004-2008 from 10 geographically diverse urban and rural areas of China.

Results $76 \%$ of men and $35 \%$ of women were current alcohol drinkers, with $33 \%$ of men and $2 \%$ of women drinking at least weekly. In men, the prevalence of weekly drinking varied by sevenfold between areas (from $7 \%$ to $51 \%$, age-adjusted), and was highest at age $40-49$ years, among those with no formal education, and among regular smokers and tea-drinkers. Among weekly drinkers, the estimated median consumption was $244 \mathrm{~g} /$ week in men and $68 \mathrm{~g} /$ week in women. Most alcohol consumption involved strong spirits, although this varied somewhat by area and there was a trend towards increased beer consumption among younger people. In male drinkers, $37 \%$ ( $12 \%$ of all men) reported regular binge drinking (ie, $>60 \mathrm{~g}$ alcohol in one session), and the prevalence was highest in younger men. Most drinkers (86\%) usually drank with meals, and a fifth reported flushing/dizziness after drinking.

Conclusion The prevalence and pattern of drinking in China show strong regional and socio-demographic variation. The health-related effects of alcohol consumption among study participants are now being monitored.

\section{P2-190 WITHDRAWN}

\section{P2-191 URBAN MIGRATION MODULATES THE EFFECT OF BODY MASS INDEX ON BLOOD PRESSURE}

doi:10.1136/jech.2011.142976j.25

1,2 J Miranda, ${ }^{* 1,3}$ A Bernabe-Ortiz, ${ }^{1,4} \mathrm{R}$ Gilman, ${ }^{5,6} \mathrm{~L}$ Smeeth. ${ }^{1}$ CRONICAS, Center of Excellence in Chronic Diseases, Universidad Peruana Cayetano Heredia, Lima, Peru ${ }^{2}$ Department of Medicine, School of Medicine, Universidad Peruana Cayetano Heredia, Lima, Peru; ${ }^{3}$ Public Health and Administration School, Universidad Peruana Cayetano 
Heredia, Lima, Peru; ${ }^{4}$ Department of International Health, Johns Hopkins Bloomberg School of Public Health, Baltimore, Maryland, USA; ${ }^{5}$ Department of Epidemiology and Population Health, London School of Hygiene and Tropical Medicine, London, UK; ${ }^{6}$ Área de Investigación y Desarrollo, A.B. PRISMA, Lima, Peru

Objective This study aims to describe the relationship between body mass index (BMI) and blood pressure in three distinct Peruvian populations.

Methods Three population groups were recruited: Rural (born and remained in Ayacucho), Migrant (born in Ayacucho and migrated to Lima), and Urban (born in Lima). Systolic blood pressure (SBP) and diastolic blood pressure were measured using oscillometric devices (Omron M5-i, Japan) and standardised techniques. BMI was calculated from standardised measurements. ANOVA was used to test differences between groups. Multi-variable linear regression was used to describe the relationship between BMI and blood pressure, adjusting for potential confounders.

Results SBP was similar in the rural $(120.9 \pm 18.7)$ and migrant groups (119.9 \pm 16.4$)$, but higher in the urban group $(128.2 \pm 22.9)$. BMI was significantly lower in the rural group (23.2 \pm 2.7$)$, but similar in the migrant $(27.0 \pm 4.3)$ and urban groups (28.3 \pm 5.4$)$. There was a positive relationship between BMI and SBP (slope 0.81; $95 \%$ CI 0.59 to 1.03 ) after adjustment for age, sex, height and haemoglobin. A positive relationship was observed in urban residents $(0.61 ; 0.04-1.18)$, but the gradient of the relationship was steeper in the migrant group $(0.75,0.48-1.02)$. Similar results were found for diastolic blood pressure.

Conclusions The relationship between BMI and blood pressure differed between our three study populations, with blood pressure rising at lower values of BMI in migrants. Migrant population in transitional countries may be at greater risk of developing hypertension, and the effect of BMI as a predictor is not uniform in migrant and urban-born residents.

\section{P2-192 AREA OF RESIDENCE OR SOCIAL CLASS, WHICH IS THE STRONGER DETERMINANT ASSOCIATED WITH CARDIOVASCULAR RISK FACTORS AMONG PAKISTANI POPULATION? A CROSS SECTIONAL STUDY}

doi:10.1136/jech.2011.142976j.26

${ }^{3} \mathrm{~F}$ Tareen, ${ }^{2} \mathrm{~K}$ Shafique, ${ }^{1} \mathrm{~S}$ Mirza, ${ }^{*}{ }^{1} \mathrm{P}$ Vart, ${ }^{3} \mathrm{Z}$ Arain. ${ }^{1}$ Dow University of Health Sciences, Karachi, Pakistan; ${ }^{2}$ University of Glasgow, Glasgow, UK; ${ }^{3}$ Afra General Hospital, Faisalabad, Pakistan

Background Prevalence of cardiovascular disease and the risk factors associated with it are increasing globally and in particular throughout the developing world. The south Asian region is especially at risk because of the increasing prevalence of the said risk factors. The study was undertaken to investigate the impact of social class and area of residence in distribution of cardiovascular risk factors mainly hypertension and diabetes mellitus in Pakistan.

Methods A cross-sectional study on 2495 subjects aged between 30 and 75 years was conducted in the Punjab province covering both the urban and rural areas. A detailed questionnaire was completed; anthropometric measurements and blood samples from the chosen subjects were taken after a written informed consent was obtained. Participants were categorised into urban and rural, and assigned a social class by occupation. A logistic regression model was used to explore the association between social class and the area of residence

Results Overall prevalence of hypertension and diabetes was $24.21 \%$ and $16.63 \%$ respectively. Out of the total number of participants, $56.79 \%(n=1417)$ were rural area residents while $43.21 \%(n=1078)$ were urban. Urban individuals appeared significantly more likely $(p<0.001)$ to be hypertensive (OR=3.03, 95\% CI 2.41 to 4.82$)$ and more likely $(p<0.001)$ to be diabetic $(O R=1.77,95 \%$ CI 1.37 to 2.29$)$ than rural dwellers after multivariate adjustments for age, gender, BMI and social class.

Conclusions The study thus concludes that the area of residence is a more powerful determinant associated with cardiovascular risk factors as compared to social class in Pakistani population.

\section{P2-193 CONFIRMATORY FACTOR ANALYSIS OF METABOLIC SYNDROME COMPONENTS IN IRANIAN ADOLESCENTS: TEHRAN LIPID AND GLUCOSE STUDY}

doi:10.1136/jech.2011.142976j.27

${ }^{2} \mathrm{M}$ Sanjari, ${ }^{2} \mathrm{~A}$ Mirzazadeh, ${ }^{*}$ S Z Asl, ${ }^{1} \mathrm{~N}$ Saadat, ${ }^{1} \mathrm{M}$ Tohidi, ${ }^{1} \mathrm{~F}$ Azizi. ${ }^{1}$ Research Institute for Endocrine Sciences, Shahid Beheshti University of Medical Sciences and Metabolic Disease Research Center, Tehran, Iran; ${ }^{2}$ Physiology Research Center, Kerman University of Medical Sciences, Kerman, Iran

Introduction There is no applicable model for identifying the metabolic syndrome in adolescent populations. The aim of this study was to identify the most components of risk variables associated with metabolic syndrome in Iranian adolescents.

Methods Anthropometry, blood pressure and biochemical measurements were assessed in a population-based study of 1307 Iranian adolescents (563 boys and 744 girls) aged 8-11 years in two phases, 1999-2001 and 2005-2007. Exploratory and confirmatory factor analysis was conducted using SPSS version. 15.

Results A two-factor structure was identified accounting for $55.8 \%$ and $53.8 \%$ of variance for boys and girls, respectively. The factor loadings for boys (and girls) in study round one two were 0.87 (0.84). 0.88 (0.82), 0.67 (0.63), 0.75 (0.8) and -0.80 (0.81) for systolic blood pressure (SBP), diastolic BP, waist circumference (WC), triglycerides (TG) and high-density lipoprotein (HDL) respectively. In phase two, the factor loadings for boys (and girls) were 0.74 (0.86), 0.76 (0.83), 0.72 (0.43), 0.75 (0.74), -0.72 (-0.77) for SBP, DBP, WC, TG and HDL, respectively. Fasting blood sugar had a loading factor of 0.47 only in boys in study round two. The twofactor model fit the data significantly in both study rounds by sex (Comparative fit index: 0.77-0.97).

Conclusion The two-factor model could be used for determining metabolic syndrome in adolescents in Iran; these two factors are blood pressure, and lipid profile/obesity.

\section{P2-194 HIGH PREVALENCE OF METABOLIC SYNDROME AMONG JAPANESE IMMIGRANT IN SOUTH BRAZIL}

doi:10.1136/jech.2011.142976j.28

${ }^{1}$ S Mizushima, ${ }^{*}{ }^{2}$ E Moriguchi, ${ }^{1} \mathrm{~N}$ Morikawa, ${ }^{3} \mathrm{Y}$ Imamatsu, ${ }^{3} \mathrm{E}$ Tadaka. ${ }^{1}$ Yokohama City University Graduate School of Medicine, Yokohama, Japan; ${ }^{2}$ Federal University of Rio Grande do Sul, Porto Alegre, Brazil, ${ }^{3}$ Yokohama City University Graduate School of Nursing, Yokohama, Japan

Introduction Prevalence of obesity and related risk factors, recognised as Metabolic Syndrome (MetS), are influenced by environmental factors including diets and physical activities, which immigrant study can show evidence.

Objectives To assess prevalence of obesity and its related risk factors among Japanese immigrant population in South Brazil compared with representative Japanese data in Japan.

Methods In August 2010, from 12 scattered colonies of Japanese immigrants in Santa Catalina and Rio Grande do Sul States, in Brazil, 274 immigrants were recruited. MetS was determined, according to Japanese Guideline, waist circumference $85 \mathrm{~cm}$ or over for males and $90 \mathrm{~cm}$ or over for females, with at least 2 accumulated related risk factors such as hypertension, high blood sugar, high triglyceride and low HDL cholesterol. Prevalence were compared 